\title{
Knowledge and attitude of nursing staff on baby-friendly Hospital Initiative practices in a Tertiary Hospital
} Mathema S $\mathrm{S}^{\mathrm{iD} \otimes}$, Shrestha S $\mathrm{S}^{\mathrm{iD}}$

${ }^{1}$ Smriti Mathema, Assistant Professor; ${ }^{2}$ Sabina Shrestha, Lecturer; Department of Pediatrics, Kathmandu Medical College Teaching Hospital, Kathmandu, Nepal.

\begin{abstract}
Background: Within a hospital setting, health professionals play a key role in providing the right information on breastfeeding and often this source are the nurses who have the primary opportunity to counsel, support and educate mothers on breastfeeding. The Baby Friendly Health Initiative (BFHI) has helped to motivate facilities providing maternity and newborn services worldwide to better support breastfeeding.

Objective: The aim of this study was to help identify the knowledge gaps on the BFHI practices among nursing staff in a tertiary care center in Kathmandu.

Methodology: A total of 27 nursing staff who encounter pregnant women admitted in the hospital up until post-delivery discharge were selected for the study. Data on knowledge of baby-friendly hospital initiative and attitude towards breastfeeding practices was collected using a structured interview between October and December 2019. Statistical analysis was done using SPSS version 20.

Results: Only $41 \%$ were acquainted with the ten steps to successful breastfeeding of BFHI, out of whom more than half (55\%) had a good level of knowledge. Majority of the participants showed a fairly correct attitude towards BFHI practices, except for the belief that there is no harm in introducing pre-lacteal feeds to babies. The opinion on demand feeding and the time of introduction of complementary feeding seemed to vary.

Conclusion: There is an urgent need to re-evaluate the present knowledge of our nursing staff and to ensure they impart the right information. The only way to achieve this is through advocacy and training to promote, support and protect breastfeeding.
\end{abstract}

Key words: BFHI practices; Breastfeeding; Knowledge; Nurses

\section{INTRODUCTION}

P reastfeeding provides adequate nutrition at the right conditions for the human infant and is

Access this article online

Website: www.jkmc.com.np

DOI: https://doi.org/10.3126/jkmc.v9i2.35528

HOW TO CITE

Mathema S, Shrestha S. Knowledge and attitude towards baby friendly health initiative among nurses working in a tertiary care hospital. J Kathmandu Med Coll. 2020;9(2):87-91.

Address for correspondence

Dr. Smriti Mathema

Assistant Professor, Department of Pediatrics,

Kathmandu Medical College Teaching Hospital, Kathmandu, Nepal.

E-mail: smritimathema@gmail.com

Copyright ๔ 2020 Journal of Kathmandu Medical College (JKMC) ISSN: 2019-1785 (Print), 2091-1793 (Online) an important aspect of child survival'. As per World Health Organization (WHO) recommendations, correct breastfeeding practices involve timely initiation of breastfeeding, exclusive breastfeeding for the first six months, and continued breastfeeding with appropriate complementary feeds till two years and beyond ${ }^{2}$. Despite well-documented benefits for mother and child and the cost-effectiveness of breastfeeding, globally only $37 \%$ of infants are exclusively breastfed in their first six-months of life ${ }^{3}$. Pre-lacteal feeding, cultural myths, workplace-related issues and aggressive marketing of commercial milk formula are some identified barriers to early initiation of exclusive breastfeeding and also are important contributory factors to the failure of continuation of breastfeeding beyond six months of age.

In spite of the proven benefits, only two-thirds of mothers in Nepal are reported to exclusively breastfeed their infants in the past 24 hours $(66.1 \%)^{4}$. The prevalence of exclusive breastfeeding in early infancy in Nepal may be in the decline, as indicated by a reduction from about 
70 to $66 \%$ between consecutive Demographic Health Surveys (DHS) from 2011 to $2016^{4}$. Many challenges might have contributed to the decline of the rates ${ }^{3}$ requiring interventions to ensure adequate support for breastfeeding, including in antenatal care, postpartum care, communities, and workplaces, as well as adequate maternity protection and code legislation.

Within a hospital setting, health professionals play a key role in providing breastfeeding support to new mothers and providing them with the right information in a respectful, positive, and supportive environment ${ }^{5}$. And, often the primary sources of information are the nurses in the postnatal wards who have the opportunity to counsel and educate new mothers on breastfeeding. In recognition of that, the Baby Friendly Health Initiative (BFHI) was birthed as a global campaign in 1991 and since then has helped to motivate facilities providing maternity and newborn services worldwide to better support breastfeeding ${ }^{6}$.There is substantial evidence of significant improvement of breastfeeding rates by the implementation of the Ten Steps to Successful Breastfeeding (the Ten Steps) ${ }^{7}$. However, research shows that a number of hospitals designated years ago as babyfriendly in Nepal have not maintained practices that reflect this status ${ }^{8}$. Strong adherence to the ten steps by the health facility is suggested to contribute to improved breastfeeding results after discharge of mother and child. The aim of this study is to help identify the knowledge gaps on this BFHI among nursing staff in a tertiary care center in Kathmandu.

\section{METHODOLOGY}

The study was conducted in Kathmandu Medical College Teaching Hospital (KMCTH), Sinamangal, Kathmandu between October and December 2019. Using institutional ethnography, the institutional processes included all nursing staff who encounter pregnant women admitted in the hospital up until the discharge of new mothers and their babies. Therefore, nursing staff working in the antenatal ward, postnatal ward, private wards, and the Neonatal Intensive Care Unit (NICU) of this hospital were included in the study. A one-on-oneface-to-face interview using a self-constructed structured questionnaire was conducted within the hospital premises during off-duty hours. Participants were briefed about the purpose of the study and participation was voluntary.

A purposeful sampling of nurses who oversee breastfeeding was done and a total of 27 nurses working in the mentioned wards of the hospital were selected for the interview. They were included in the study based on their voluntary agreement to participate and the interview was conducted as per their availability during the period of the study of 10 weeks. An informed consent was obtained from each participant and each interview was recorded.

The outline of the interviews focused on questions to help identify the gap in knowledge of ten steps of BFHI (Table 1) and their attitude towards breastfeeding practices which influence the implementation of these steps. The steps of the BFHI that have been implemented in recent years in form of programs or training as per the experience of the participants were identified, along with their perceptions on its feasibility in the near future. Statistical analysis was done using Statistical Package for the Social Sciences (SPSS) version 20.

\section{RESULTS}

The response rate of the total number of questions asked at the interview was 98.6 percent. The respondents were all females and they varied widely in their number of years of experience. The characteristics of participants are presented in Table 2. The median age of the nursing staff was 28.7 and the median years of job experience was 4.9.

Only $41 \%$ (11 out of the total 27 respondents) were acquainted with the ten steps to successful breastfeeding of the BFHI, while the remaining 16 nursing staff were hearing the term for the very first time during the interview. Out of 11 nurses who were familiar with BFHI more than half $(55 \%)$ had good, one-third $(36 \%)$ had satisfactory and 1 (9\%) had a poor level of knowledge (Table 3). Table 4 shows a fairly correct attitude towards $\mathrm{BFHI}$ practices, except for the belief that there is no harm in introducing pre-lacteal feeds to babies. However, the opinion on demand feeding and the appropriate time of introduction of complementary feeding seemed to vary equally. 


\section{Table 1: Ten Steps to Successful Breastfeeding ${ }^{9}$}

\section{Critical management procedures}

1. a. Comply fully with the International Code of Marketing of Breast-milk Substitutes and relevant World Health Assembly resolution.

b. Have a written infant feeding policy that is routinely communicated to staff and parents.

c. Establish ongoing monitoring and data-management systems.

2. Ensure that staff has sufficient knowledge, competence and skills to support breastfeeding.

\section{Key clinical practices}

3 Discuss the importance and management of breastfeeding with pregnant women and their families.

Facilitate immediate and uninterrupted skin-to-skin contact and support mothers to initiate breastfeeding as soon as possible after birth.

5 Support mothers to initiate and maintain breastfeeding and manage common difficulties.

6 Do not provide breastfed newborns any food or fluids other than breast milk, unless medically indicated.

7 Enable mothers and their infants to remain together and to practice rooming-in 24 hours a day.

8 Support mothers to recognize and respond to their infants' cues for feeding.

9 Counsel mothers on the use and risks of feeding bottles, teats and pacifiers.

10 Coordinate discharge so that parents and their infants have timely access to ongoing support and care

Table 2: Characteristics of Participants

\begin{tabular}{llcc}
\hline Variables & & Total $(\mathbf{n}=\mathbf{2 7})$ \\
\hline & $20-30$ & 18 \\
Age (years) & $30-40$ & 6 \\
& $40-50$ & 3 & 9 \\
Education & Proficiency Certificate Level (PCL) in Nursing & 18 & 11 \\
& Bachelor's of Nursing & $0-2$ & 8 \\
Years of Job Experience & $3-5$ & 3 & 5
\end{tabular}

Table 3: Knowledge of BFHI practices

\begin{tabular}{lcc}
\hline & Total $(\mathbf{n = 2 7})$ \\
\hline Not acquainted with BFHI practices & 16 \\
\hline Acquainted with BFHI practices & Knowledge Level & 11 \\
& Good & 6 \\
& Adequate & 1 \\
\hline
\end{tabular}

Table 4: Nurses attitude towards BFHI practices

\begin{tabular}{|cc}
\hline Attitude & Total (n=27) \\
\hline Believes babies should not be given pre-lacteal feeds & $(22.2 \%)$ \\
\hline Believes in rooming-in & $24(88.9 \%)$ \\
\hline Believes in demand feeding & $18(66.7 \%)$ \\
\hline Believes complementary feeding should not be started prior to six months of age & $17(63 \%)$ \\
\hline Believes feeding bottles are harmful & $22(81.5 \%)$ \\
\hline Does not believe in the use of pacifier & $21(77.8 \%)$ \\
\hline
\end{tabular}




\section{DISCUSSION}

As part of breastfeeding promotion, hospitals are expected to promote exclusive breastfeeding. Furthermore "Breastfeeding Week" is celebrated in the first week of August annually to emphasize the importance of exclusive breastfeeding. Despite these efforts, exclusive breastfeeding rate still remains low, especially so in urban settings. A significant finding is that mothers tended to exclusively breastfeed longer if they received breastfeeding advice immediately after childbirth ${ }^{10}$. As originally recommended in the ten steps of $\mathrm{BFHI}$, these messages and support include advice on early initiation, no other food items but breastmilk, keeping infant and mother together, breastfeeding on demand, no teats or pacifiers, where to go when problems occur, and skill on position, holding newborn and latching. This form of breastfeeding support in the hospital setting mainly comes from nursing staff working in the antenatal and postnatal wards, along with those working in the NICU. A Brazilian study also observed a longer exclusive breastfeeding duration among mothers who received breastfeeding support in hospital ${ }^{11}$.

However, our study demonstrated a lack of awareness of some of the major recommended practices in the hospital that will promote and sustain breastfeeding. There was a huge knowledge gap even in presumably universally accepted facts such as pre-lacteal feeds being harmful to the newborn. Information such as on the use of pacifiers and feeding bottles were rightly believed to be best avoided, but there seems to be a constant need for reinforcement through advocacy and training.

Out of the respondents, only $66.7 \%$ believed in demand feeding and the percentage of those who believed complementary feeding should not be started prior to the recommended age of six months was only $63 \%$. As highlighted by previous similar studies ${ }^{12}$, the lack of awareness of breastfeeding practices among health professionals is a major obstacle to proper breastfeeding practices. Breastfeeding promotion in the form of advice and skill support is known to show positive effects on breastfeeding indicators ${ }^{12,13}$. Most importantly, critical evaluation of the effectiveness of the individual steps of BFHI, not only as to the hospital-based breastfeeding initiation and exclusivity rates but more importantly to the long-term breastfeeding rates (length of duration and degree of exclusivity), became the desired end point ${ }^{14}$.

\section{CONCLUSION}

Despite the fact that Nepal implemented its babyfriendly hospital initiative in 1991, no evaluation has been undertaken on its effect. There is an urgent need to re-evaluate the present knowledge of our nursing staff and to ensure they impart the right information. The only way to achieve this is through advocacy and training to promote, support and protect breastfeeding.

Conflict of interest: The author declares that no competing interest exists.

Financial Disclosure: None

\section{REFERENCES}

1. Egbuonu I, Ojukwu JU. Infant feeding. In: Azubuike JC, Nkanginieme KEO, editors. Paediatrics and Child health in a tropical region. African educational services 2007;224-239.

2. World Health Organization, United Nations Children's Fund. Global strategy for infant and young child feeding. 2003.[Full Text]

3. World Health Organisation\& UNICEF (2017). Global Breastfeeding Scorecard: Tracking Progress for Breastfeeding Policies and Programmes. New York: UNICEF. [Full Text]

4. Ministry of Health, New ERA, ICF International Inc. Nepal Demographic and Health Survey 2016. Kathmandu: Ministry of Health; 2017.[Full Text]

5. Lutter CK, Perez-Escamilla R, Segall A, Sanghvi $T$, Teruya K, Wickham C. The effectiveness of a

hospital-based program to promote exclusive breast-feeding among low-income women in Brazil. Am J Public Health. 1997 Apr;87(4):659-63. [DOIFull TextPubMed]

6. World Health Organization, United Nations Children's Fund. The Baby-friendly Hospital Initiative: monitoring and reassessment: tools to sustain progress. Geneva: World Health Organization; 1991 (WHO/NHD/99.2). [Full Text]

7. World Health Organization \& United Nations


promoting and supporting breast-feeding: the special role of maternity services / a joint WHO/ UNICEF statement. World Health Organization.[Full Text]

8. Subedi, N. Baby-Friendly Hospital Initiative: Situation in Nepal. Health Prospect. 2012;11:53-4. [DOIFull Text] 
9. World Health Organisation\& UNICEF (2018). Implementation guidance: protecting, promoting and supporting breastfeeding in facilities providing maternity and new-born services - the revised Babyfriendly Hospital Initiative. Geneva: World Health Organisation.[Full Text]

10. Khanal V, Lee AH, Karkee R, Binns CW. Postpartum Breastfeeding Promotion and Duration of Exclusive Breastfeeding in Western Nepal. Birth. 2015 Dec;42(4):329-36. [DOIPubMedFull Text]

11. Vieira TO, Vieira GO, de Oliveira NF, Mendes CMC, Guigliani ERJ, Silva LR. Duration of exclusive breastfeeding in a Brazilian population: new determinants in a cohort study. BMC Pregnancy
Childbirth. 2014 May 26; 14:175. [DOIFull TextPubMed]

12. Hawkins SS, Stern AD, Baum CF, Gillman MW. Evaluating the impact of the Baby-Friendly Hospital Initiative on breast-feeding rates: a multi-state analysis. Public Health Nutr. 2015 Feb;18(2):189-97. [DOIPubMedFull Text]

13. Venancio SI, Saldiva SRDM, Escuder MML, Giugliani ERJ. The Baby-Friendly Hospital Initiative shows positive effects on breastfeeding indicators in Brazil. J Epidemiol Community Health. 2012 Oct;66(10):914-8. [DOIFull TextPubMed]

14. Eidelman Al. A Critical Review of the Baby-Friendly Hospital Initiative Is in the Works. Breastfeeding Medicine. Nov 2018;13(9):557-8. [ DOIFull Text] 